\title{
Short to Mid-Term Term Surgical Outcome Study with Posterior Only Approach on Tuberculous Spondylodiscitis in an Elderly Population
}

\author{
Manish Kothari, Kunal Shah, Agnivesh Tikoo, Abhay Nene \\ Department of Spine, Wockhardt Hospital, Mumbai, India
}

\section{Study Design: Retrospective study.}

Purpose: To study short to mid-term outcome of surgically managed elderly patients of tuberculous spondylodiscitis with posterior only approach in terms of decision making and challenges in treatment, choice of implants and outcomes.

Overview of Literature: Tuberculous spondylodiscitis in the elderly is increasing due to longer survival rates. It presents with varied clinical manifestations needing surgical management. Management in tuberculous spondylodiscitis has been scarcely reported in the elderly, with a paucity of data on the choice of implants and approach.

Methods: Sixteen patients (five males, 11 females) older than 70-years-of-age culture and/or histopathology proven tuberculous spondylodiscitis were included in the study. All patients were operated using a single posterior approach. Pedicle screw with rods (PS/ rods) or spinal loop with sublaminar wires (SL/SLW) were used for fixation. Clinical and surgical details were recorded. Sagittal correction achieved postoperatively and loss of correction at follow-up were noted.

Results: The mean age was 73.6 years (range, 70 to 80 years). The mean follow up was 44.5 months (range, 24 to 84 months). The mean immediate postoperative correction of sagittal deformity was 11.3 degrees; this correction was lost by a mean of 3.1 degrees at last follow-up. All 10 patients with deficit showed neurological recovery and all but one of the seven non-walkers were capable of independent ambulation at follow-up. Patients with SL/SLW and PS/rods had similar radiological outcome at final follow up.

Conclusions: Operative management gives satisfactory results in elderly patients with tuberculous spondylodiscitis. The posterior approach provides adequate exposure for decompression and rigid fixation, providing satisfactory clinical and radiological outcomes. SSL/SLW and pedicle screw rod construct both give similar radiological results if used appropriately in patients.

Keywords: Elderly; Tuberculosis; Posterior surgery; Outcome

\section{Introduction}

Tuberculosis of the spine is most common manifestation of skeletal tuberculosis. It commonly affects younger people but can affect any age group [1]. Tuberculosis is an emerging trend in the elderly, especially because of their prolonged survival. At the same time, spinal affection has also increased with a varied spectrum of presentation $[2,3]$.

Conservative treatment is usually the first line of treatment for tuberculous spondylodiscitis in any age group. Elderly patients face a plethora of challenges with con-

\footnotetext{
Received Jun 26, 2015; Revised Aug 9, 2015; Accepted Aug 9, 2015

Corresponding author: Kunal Shah

Wockhardt Hospital, South Mumbai, 1877, Dr.Anand Rao Nair Road, Near Agripada Police Station,

Mumbai Central (E), Mumbai- 400 011, Maharastra, India

Tel: +91-99-3073-1911, E-mail: orthokunal@yahoo.com
} 
servative treatment in view of complications of bed rest, associated co-morbid conditions and disability [4]. This prompts operative treatment in certain situations.

Spinal surgeries in elderly are associated with higher complications because of poor bone stock, co-morbid conditions and compromised general ability to cope with surgery [5,6]. A quick, effective and safer approach to optimally target the pathology and aid early mobilization in the elderly is needed.

Surgical treatment in tuberculosis spondylodiscitis is scarcely reported, with only a few studies on surgical management in the elderly $[7,8]$. Furthermore there is paucity of data on type of approach and choice of implants in elderly. The single posterior approach is well established in younger patients with tuberculous spondylodiscitis [9]. However, its role in elderly patients is not validated.

This study was undertaken to assess surgically managed elderly tuberculous spondylodiscitis patients with the single posterior approach in terms of decision making and challenges in treatment, choice of implants and outcomes.

\section{Materials and Methods}

We retrospectively analyzed 276 consecutive spondylodiscitis patients managed in our spine clinic from 2005 to 2013. We identified 16 patients over 70-years-of-age who had been managed surgically with a minimum of 2 years follow-up. All patients had culture and/or histopathology proven tuberculous spondylodiscitis from samples taken either preoperatively or during surgery. Patients younger than 70 years, on empirical anti-tubercular drugs and with negative histopathological diagnosis were excluded. All patients underwent single posterior approach surgery. The data was collected from hospital records via an institutional data retrieval system and the surgeon's own database. Data was evaluated by an independent observer. Clinical data included spectrum of symptoms, co-morbidities and indication of surgery. Neurology was assessed using the Frankel grading system. The surgical details included time for induction, blood loss, operative time, instrumentation details, number of levels fixed and method of decompression. The indications for surgery were intolerable instability type back pain and or leg pain with failure of medical management and neurological deficit (either profound or not improving with medical therapy). Table 1 shows details of patients operated.

\section{Surgical procedure}

Under general anaesthesia the patients were positioned prone. Posterior midline exposure was used. In cases with pedicle screw fixation, pilot holes were made using the freehand technique. In cases with spinal loop fixation, the sublaminar wires were passed above and below the level of laminectomy. Laminectomy was done according to the extent of neural compression. In cases with no deficit, only a hemilaminectomy was done to preserve the posterior element integrity. The epidural abscess was drained and granulation tissue was carefully separated from the dural layer and excised. On the side of maximum compression, as seen on axial magnetic resonance imaging (MRI), the pedicle below the involved disc space was removed using a Leksel rongeur to the level of the posterior cortex of the vertebral body. Necrotic bone and granulation tissue in the anterior column were curetted out. The debridement was conservative and limited to necrotic bone and tissue only to minimize the length of the anterior column defect. Additionally, posterior column shortening was done to close the defect and correct the kyphosis. Local autograft was used to fill the anterior column defect. An allograft was added when the autograft was not sufficient. Although we routinely use metal cages in younger patients, we did not use metal cages in this population to reduce the operative time, blood loss and morbidity of surgery. We also did not use iliac crest strut graft for the same reasons, except in one patient with a larger defect. When the anterior defect and kyphosis was limited to one disc height, only transpedicular decompression was done to excise the offending lesions compressing the cord. In the lumbar spine we used the transforaminal approach for anterior debridement and grafting. Fixation was achieved using segmental instrumentation. In general for the earlier patients spinal loop with sublaminar wires (SL/SLW) construct was used and 2008 onwards pedicle screw rod (PS/rod) construct was used. One patient underwent noninstrumented decompressive laminectomy and biopsy, as he had presented with predominantly epidural collection and posterior element involvement.

\section{Postoperative protocol}

Anti-tubercular drugs were given for one year. Postoperatively patients were mobilized as tolerated with orthotic support (in a few cases). 


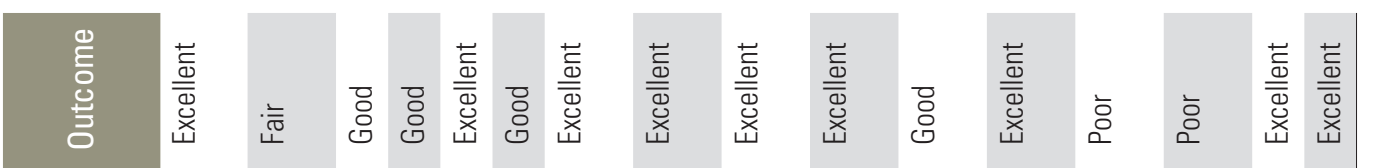
Fing

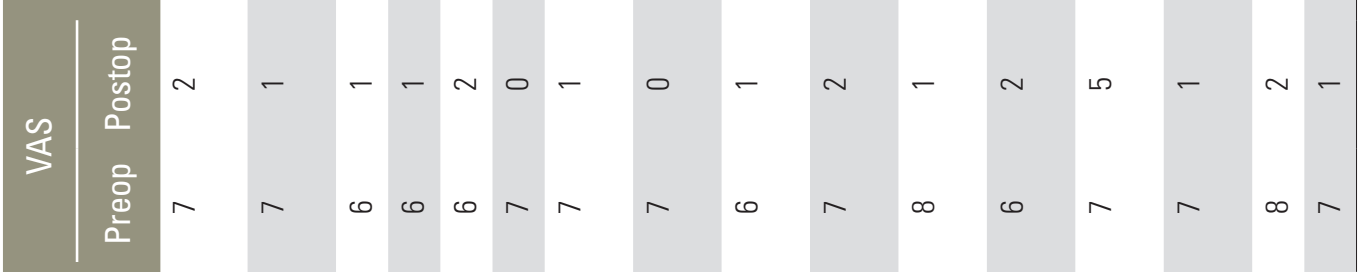

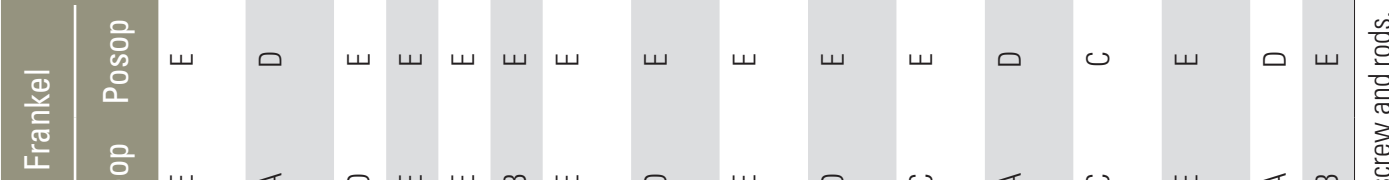

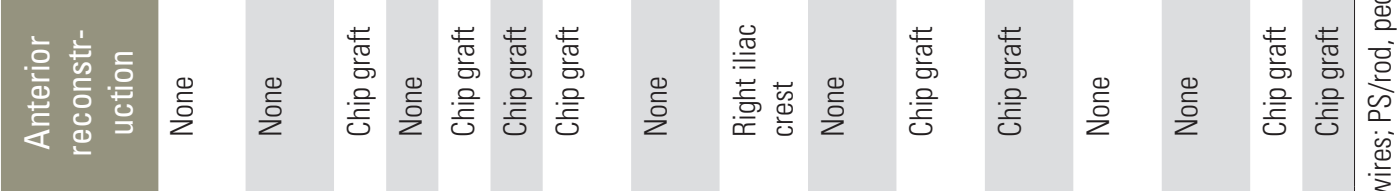

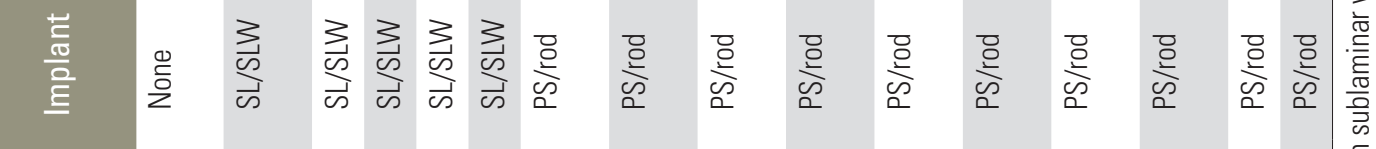

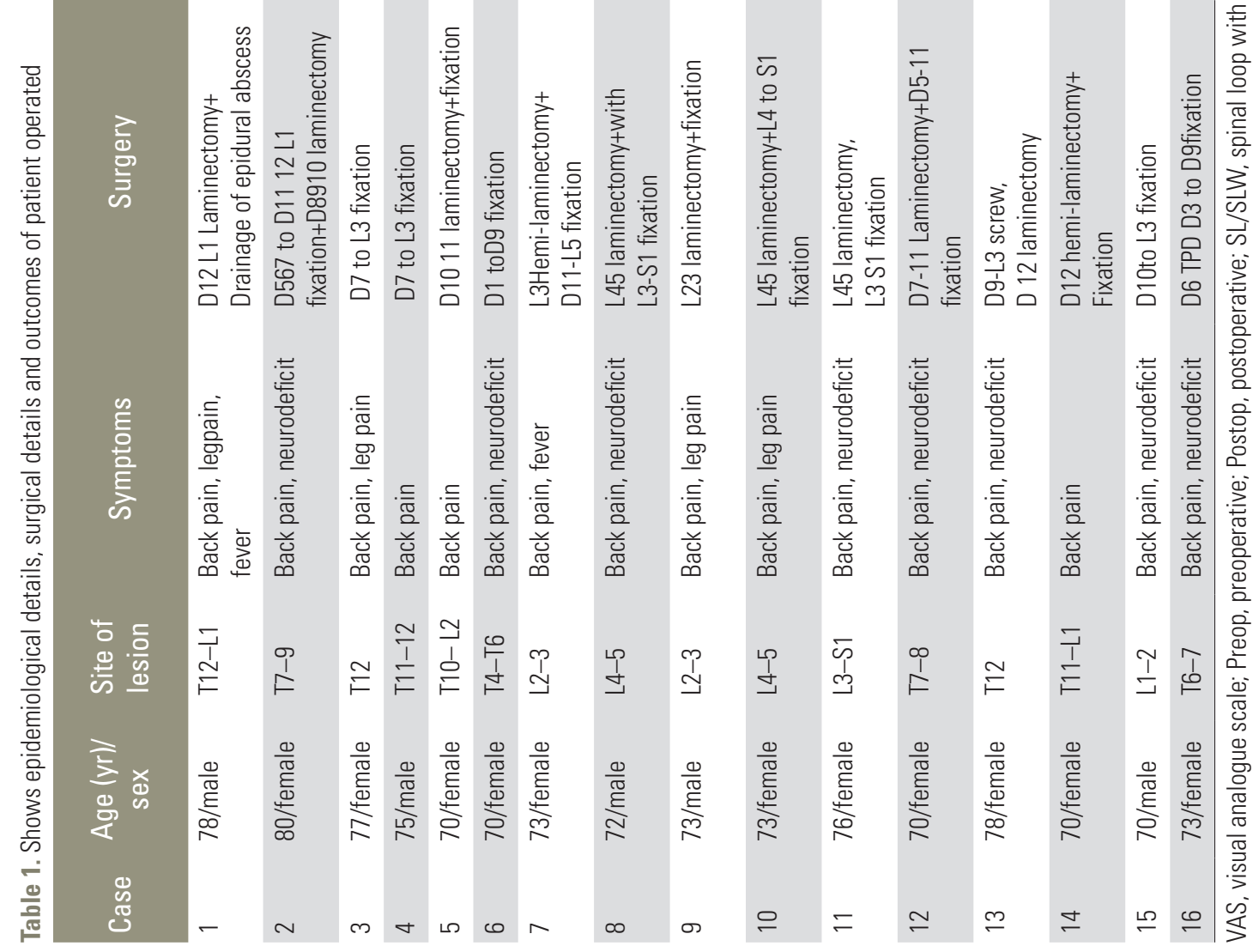




\section{Follow-up}

The patients were followed up at 6 weeks, 3 months, 6 months and yearly thereafter. Pain status was evaluated using a visual analogue scale (VAS) at each visit. Radiographs were taken at each visit to check the status of implants and fusion. MRI was done at 3 months, one year and if required at 2-year intervals to check for healing. Healed was judged to have occurred when MRI showed resolution of disease with fatty marrow replacement. In cases where sublaminar wires with spinal loop rectangle were used, MRI was not possible since they were made of stainless steel. In such cases, healing was declared based on clinical response, laboratory markers and radiographs. Computed tomography scan was used to follow-up and soft tissue resolution was studied.

The outcomes were classified as excellent (return to previous activities with little or no complaints), good (return to previous activities, but occasionally with recurrent back pain after activities), fair (reduction of previous activities necessary) and poor (no return to previous activities possible) [10].

\section{Results}

\section{Demographics}

There were five males and 11 females with a mean age of 73.6 years (range, $70-80$ years). The mean follow up was 44.5 months (range, $24-84$ months).

\section{Levels}

The thoracic spine was involved in four patients, thoracolumbar junction was involved seven patients and lumbar spine was involved five patients. There were five patients with three or more contiguous vertebrae involved. One patient had four vertebrae involvement and another one had five contiguous vertebrae involved. Multilevel involvement was seen exclusively in females. Average number of vertebrae involved was 2.5 .

\section{Co-morbidities}

There were 12 patients (75\%) with one or more medical co-morbidities. The average number of co-morbidities in each patient was two, with hypertension followed by dia- betes mellitus being the most common.

\section{Symptomatology}

Axial back pain was the most common symptom present in all the patients. Patients with lumbar lower lumbar levels had additional leg pain component. Constitutional symptoms were present in only two patients. The mean VAS was 6.81 (range, 6-8). Neurological deficit was present in 10 patients with three patients with Frankel A, two patients with Frankel B and $\mathrm{C}$ each and three patients with Frankel D grade.

\section{Procedure}

The average number of vertebral levels fused was 5.9 vertebrae. The mean blood loss and operative time was 535 $\mathrm{mL}$ and 147 minutes, respectively. Eight patients underwent transpedicular anterior column reconstruction with local chip graft [11] and one patient with an iliac crest graft. In cases where local bone proved to be insufficient, allografts were used. A cage was not used in any of the patients. The decision for anterior column chip graft reconstruction was made intraoperatively depending on the size of the defect after a previously described conservative debridement. Defects restricted to one disc height size typically did not require anterior reconstruction (eight patients). Larger defects were filled with local autograft (eight patients) with one patient requiring an iliac crest strut graft due tolarge defect. The mean immediate postoperative correction of sagittal deformity was 11.3 degrees. This correction was lost by a mean of 3.1 degrees at the last follow-up. The comparison of patients instrumented with SL versus PS is shown in Table 2. In the PS group, fewer levels were fused with minimal loss of sagittal correction at follow-up in both the SL and PS groups. The blood loss and operative time was not very different in both the groups. No patient had hardware failure and no patient underwent revision surgery.

\section{Patterns of healing}

The majority of patients healed with resolution of soft tissue shadows and fatty marrow replacement of the vertebrae at completion of chemotherapy (one year). One patient was a suspected to harbor a drug-resistant lesion preoperatively. Preoperatively, she had a worsening ab- 
Table 2. Shows comparison of patients instrumented with hartshil rectangle with sublaminar wire versus pedicle screw rod construct

\begin{tabular}{lccc} 
Characteristic & SL/SLW & PS/rods & $p$-value \\
Levels fused & 9 (SD 0.7) & 5 (SD1.8) & 0.01 \\
Blood loss (mL) & 530 (SD 345) & 572 (SD329) & 0.440 \\
\hline Operative time (min) & 147 (SD 28) & 156 (SD40) & 0.371 \\
Sagittal correction postop ( ${ }^{\circ}$ ) & 10.2 (SD 9.3) & 13.1 (SD7.4) & 0.371 \\
\hline Loss of correction at follow-up $\left(^{\circ}\right)$ & 3 (SD 1.6) & 3.21 (SD2.2) & 0.953 \\
\hline
\end{tabular}

SL/SLW, spinal loop with sublaminar wires; PS/rod, pedicle screw and rods; SD, standard deviation; postop, postoperative.

a) Mann Whitney U test.

Table 3. Shows radiological correction at each region

\begin{tabular}{lccccc} 
Region & Cobb preop & $\begin{array}{c}\text { Cobb immediate } \\
\text { postop }\end{array}$ & $\begin{array}{c}\text { Kyphosis } \\
\text { correction }\end{array}$ & $\begin{array}{c}\text { Cobb last } \\
\text { follow-up }\end{array}$ & $\begin{array}{c}\text { Loss of correction } \\
\text { at last follow-up }\end{array}$ \\
Thoracic $\left(^{\circ}\right)$ & 16.75 & 5.25 & 11.5 & 8.25 & 3 \\
\hline Thoraculumbar $\left({ }^{\circ}\right)$ & 15.14 & 6.18 & 8.96 & 9.85 & 3.67 \\
\hline Lumbar $\left({ }^{\circ}\right)$ & 9.4 & -5.28 & 14.68 & -2.4 & 2.88 \\
\hline
\end{tabular}

Preop, preoperative; Postop, postoperative.

scess despite 8 months of first-line medical therapy. She was operated for severe mechanical back pain with radiographic destruction. Tuberculosis mycobacteria growth indicator tube cultures were negative, but histopathology showed a tuberculous lesion. She was started on secondline drugs by the pulmonologist for 2 years, which was successful. There was one patient with persistent paraspinal abscess at 6 months follow-up. However, MRI showed healing of the vertebral lesion. The patient was declared healed, and the abscess was considered sterile residual fluid and aspirated with no recurrence. One patient had an implant back-out not requiring revision surgery.

\section{Recovery}

All the patients with deficits showed neurological recovery and all non-walkers were independent ambulators at follow-up except one (Frankel C). The preoperative Frankel distribution $A=3, B=2, C=2, D=3, E=6$ changed to $C=1, D=3$ and $E=12$ at follow-up. Nine patients had an excellent outcome, four had a good outcome, one had a fair outcome and two had poor outcome. One patient with a poor outcome was frail and had severe bronchial asthma. The other poor outcome patient had end-stage renal disease with left ventricular failure. The radiological correction as per the region is shown in Table 3. Figs. 1-3 show case 15,10 , and 4 , respectively.

\section{Discussion}

The effect of age and co-existing medical diseases on surgical results cannot be ignored. As age increases, comorbidities increase and surgical outcomes are affected. Posterior lumbar decompression and fusion surgery is a routinely performed surgery in the elderly for degenerative lumbar disease; these patients have a higher rate of major complications [12]. High morbidity and mortality has been reported in elderly patients with pyogenic spondylodiscitis operated using posterior instrumentation surgery [10]. The authors recommended that surgery should not be the first-line treatment in elderly patients with co-morbidities. Two studies on surgical treatment in elderly with tuberculous spondylodiscitis reported satisfactory outcomes $[7,8]$. Spinal tuberculosis has an insidious course of development and ultimately causes gross destruction and instability requiring major reconstructive surgeries. Patients on conservative management can present with gross instability or neurological deficit [1]. The decision to offer operative treatment should be timely and precise, especially in the elderly in view of co-morbidities and risk-benefit ratio. 

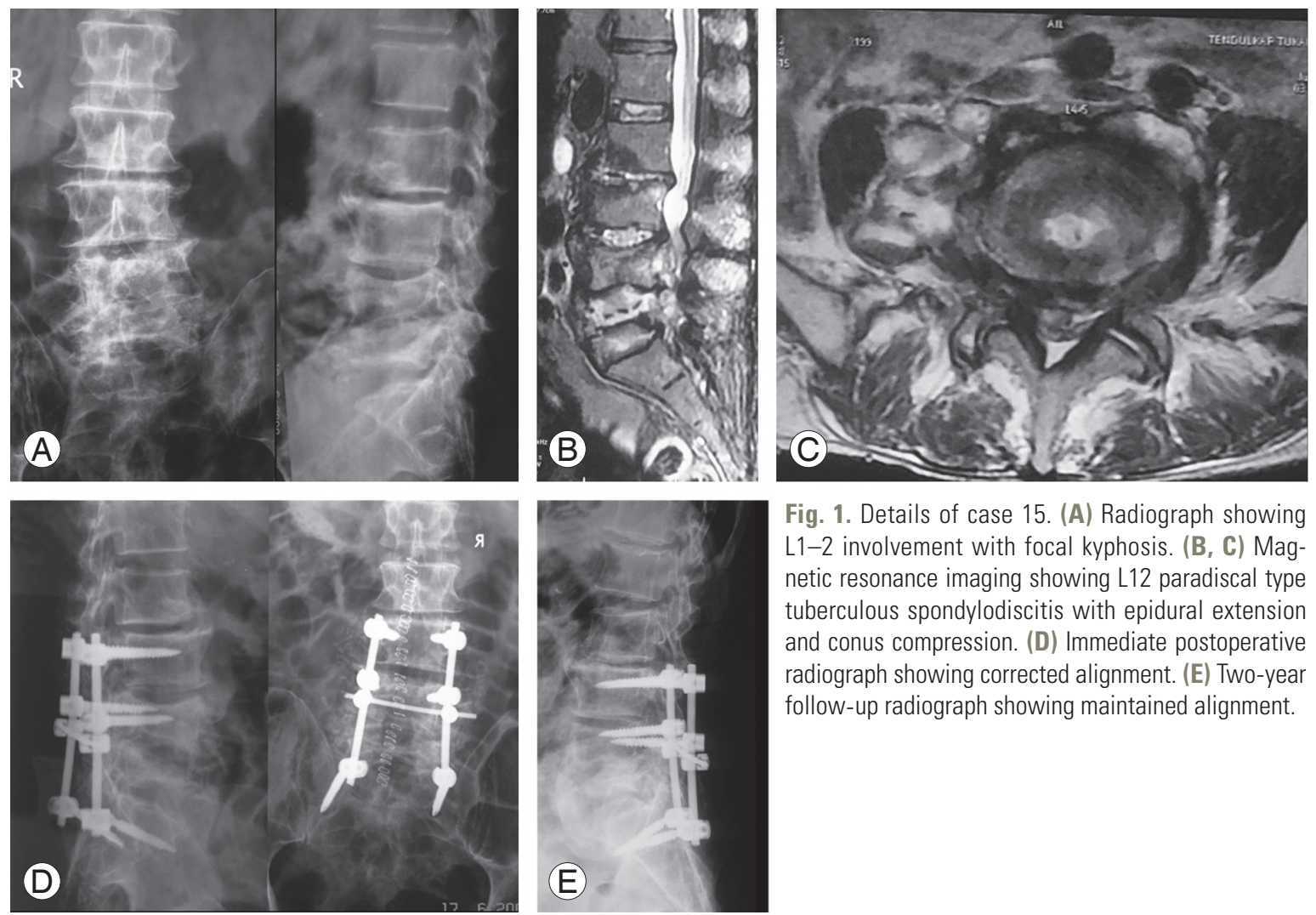

Fig. 1. Details of case 15. (A) Radiograph showing L1-2 involvement with focal kyphosis. (B, C) Magnetic resonance imaging showing L12 paradiscal type tuberculous spondylodiscitis with epidural extension and conus compression. (D) Immediate postoperative radiograph showing corrected alignment. (E) Two-year follow-up radiograph showing maintained alignment.
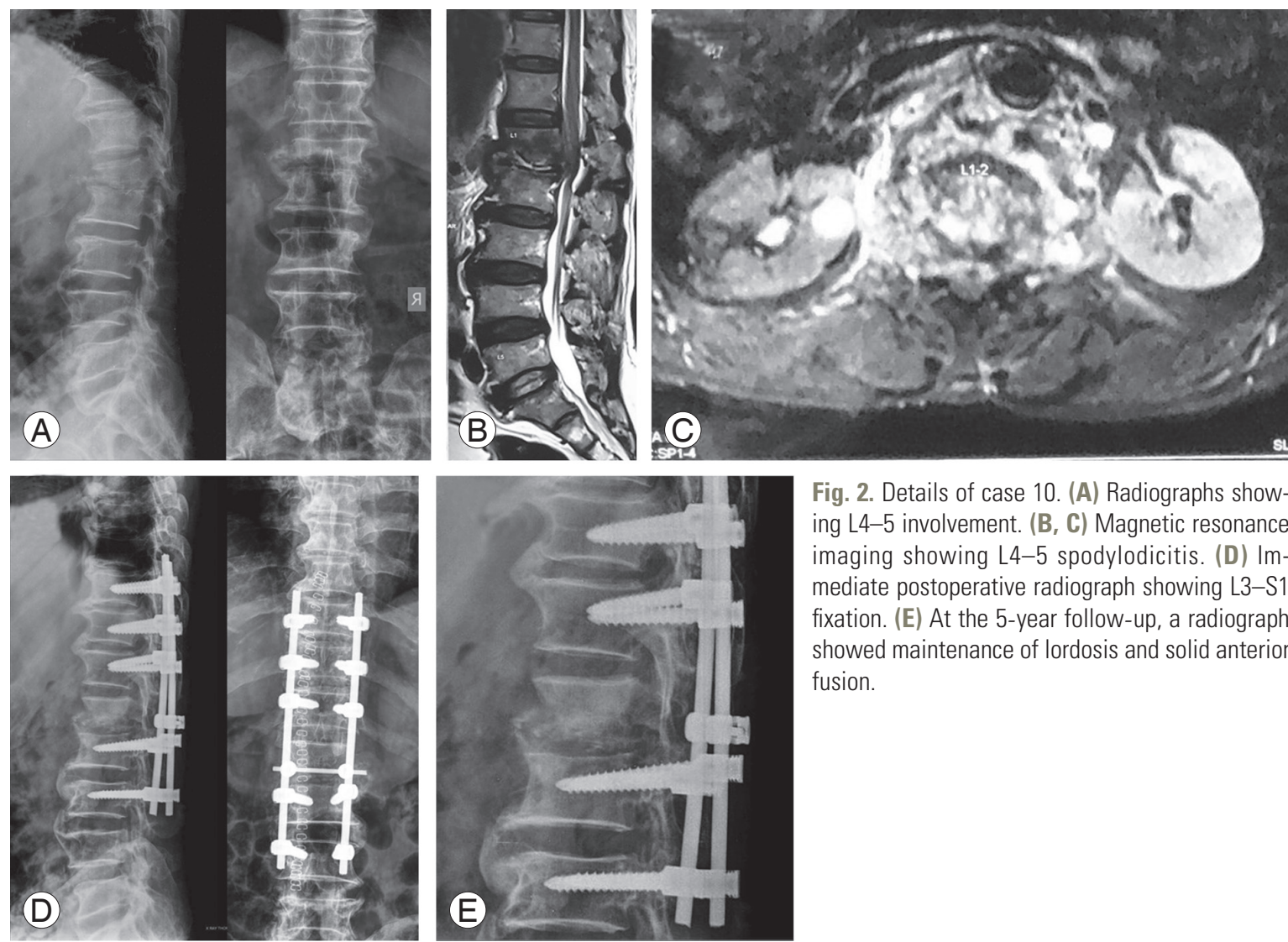

Fig. 2. Details of case 10. (A) Radiographs showing $L 4-5$ involvement. (B, C) Magnetic resonance imaging showing L4-5 spodylodicitis. (D) Immediate postoperative radiograph showing L3-S1 fixation. (E) At the 5-year follow-up, a radiograph showed maintenance of lordosis and solid anterior fusion. 

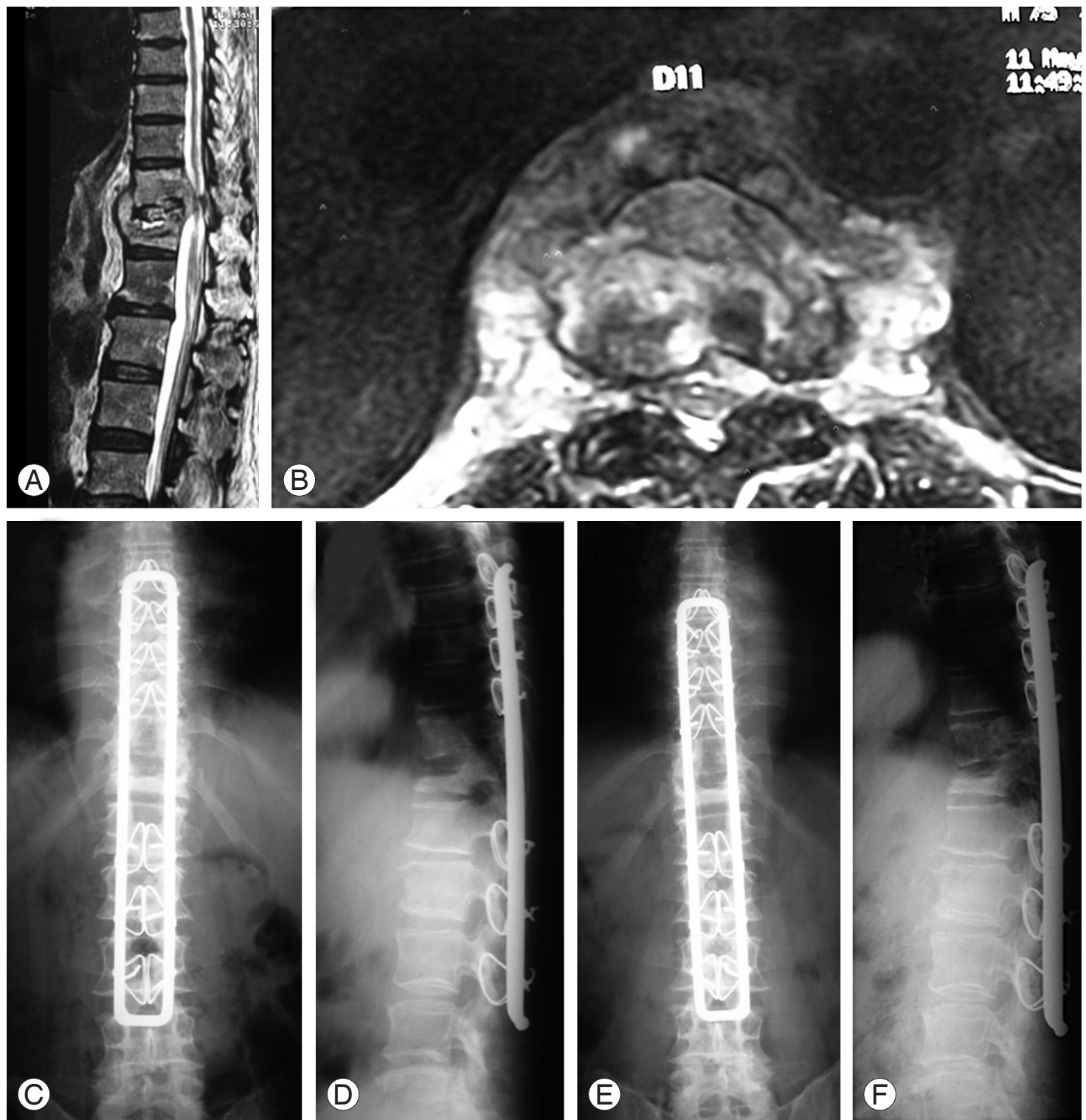

Fig. 3. Details of case 4. (A, B) Preoperative magnetic resonance imaging showing D11-12 lesion. (C, D) The patient underwent transpedicular decompression and D7 to L3 spinal loop with sublaminar wires fixation. (E, F) Two-year follow-up showing formation of lateral bridging osteophytes with mild increase in kyphosis with fusion across the posterior aspect of the vertebral body.

Perioperative challenges need to be emphasized while operating on elderly patients. Counseling in view of comorbidities, postoperative intensive care unit stay and delay in standard recovery process is required. These patients are also more prone to anesthesia-related complications. Malnutrition, anemia and dys-electrolytemia requiring multiple blood transfusion and albumin infusion should be borne in mind. Even preoperative antiosteoporosis therapy is recommended by some authors [7].

Traditionally, spinal anterior surgery is recommended to clear the tuberculous tissue. Anterior thoracic spine surgery is associated with pulmonary and other complications, even in the young [9]. Its use in the elderly requires a strong indication, especially since several studies have reported satisfactory outcomes with posterior alone surgery in tuberculosis of spine $[7,8]$. The studies compared the surgical results of combined anterior plus posterior surgery versus only posterior surgery in thoracic spine tuberculosis in the elderly. Superior clinical outcomes were evident in the posterior only surgery group. In our series, all patients with thoracic and lumbar spine involvement 
were treated with single posterior approach with satisfactory outcomes at short to mid-term follow-up.

Anterior debridement and reconstruction via the posterior approach is key for satisfactory spinal reconstruction. The transpedicular approach provides global approach to all three columns of thoracic and lumbar spine using the posterior exposure, which reduces hazards of anterior surgery, provides direct access to the spinal canal and allows the use of a variety of posterior implants [13]. This lessens the overall operative time. However, the number of levels of instrumentation remains high to provide stable construct in osteoporotic spine. Thus, the posterior only approach provides a surgeon a comfortable and targeted way of achieving adequate decompression and reconstruction with less morbidity. Also, degenerative changes in spine alter the normal anatomy and pose a risk of neurological complications [7]. The additional operative time and subsequent blood loss in an anterior approach only adds to the risk of complications in an already compromised elderly patient. Therefore, a surgeon should be aware of intra-operative challenges in treating elderly patients with spondylodiscitis.

Autologous bone graft is the gold standard for reconstruction. However, it increases procedure-related morbidity, blood loss and requires additional operative time. There is a limitation in the amount of bone graft procured in elderly patients. Also, the amount of structural stability provided by osteoporotic autografts is also doubtful [14]. We used local cortico-cancellous bone graft in majority of cases, which proved to be sufficient. In cases of large defects, the iliac crest graft was supplemented with allograft to aid fusion.

Tuberculosis is usually associated with poor living standards and social factors, which affect the elderly more than the young [1]. In such scenarios, sublaminar wires are a cheaper alternative to the pedicle screw rod construct. Also, the efficacy of sublaminar wires in the osteoporotic spine is well known [15]. However the number of levels fused and subsequent blood loss is higher in the SL/SLW group. It also has a disadvantage of being made of stainless steel and postoperative MRI is not possible. In our series, both the systems had similar operative time and radiological outcome.

Hardware failure is a known complication in the osteoporotic spine. In thoracic spine fixations, we did not have any hardware failure with spinal loop/sublaminar wire fixation or pedicle screw rod construct. As expected, the number of levels included in the construct was more in the SL/SLW construct, leading to longer incisions. The radiological results in both the fixations were similar. In the lumbar spine we encountered one hardware back out (case 3). This patient with L4-5 lesion underwent a short (L4-S1) posterolateral fusion without anterior reconstruction, which was the cause of hardware failure. Fortunately, she recovered well with expectant management over 6 months. For lumbar spine lesions, we usually achieve $360^{\circ}$ fusion by means of transforaminal lumbar interbody fusion. However, in this case anterior interbody reconstruction could not be achieved along with inadequate upper fixation levels. In a bid to save levels, the fixation was compromised upon leading to inferior fixation, demonstrating the need for longer fixations in osteoporotic spine instrumentations.

There are few shortcomings of this study. The study group is relatively smaller; however, this can be attributed to less number of patients operated in elderly age. There is no comparative group of anterior surgery alone or anterior plus posterior surgery. Long-term study is required to judge the outcome of posterior only surgery in elderly with tuberculous spondylodiscitis.

\section{Conclusions}

In conclusion, our study describes surgical outcome in tuberculous spondylodiscitis in elderly population. Operative management gives satisfactory results in very elderly patients with tuberculous spondylodiscitis. Posterior only surgery gives good clinical and radiological outcomes. Spinal loop/sublaminar wires and pedicle screw rod construct, both give similar radiological results if used appropriately in select patients.

\section{Conflict of Interest}

No potential conflict of interest relevant to this article was reported.

\section{References}

1. Tuli SM. Tuberculosis of the skeletal system: bones, joints, spine, and bursal sheaths. New Delhi: Jaypee Brothers Medical Publishers; 2004.

2. World Health Organisation. Global tuberculosis report 2014 [Internet]. Geneva: Switzerland; 2015 [cited 
2015 march 25]. Available from :http://www.who.int/ tb/publications/global_report/en/.

3. Rajagopalan S. Tuberculosis and aging: a global health problem. Clin Infect Dis 2001;33:1034-9.

4. Dittmer DK, Teasell R. Complications of immobilization and bed rest. Part 1: Musculoskeletal and cardiovascular complications. Can Fam Physician 1993; 39:1428-32.

5. Schoenfeld AJ, Ochoa LM, Bader JO, Belmont PJ Jr. Risk factors for immediate postoperative complications and mortality following spine surgery: a study of 3475 patients from the National Surgical Quality Improvement Program. J Bone Joint Surg Am 2011; 93:1577-82.

6. Fu KM, Smith JS, Polly DW Jr, et al. Correlation of higher preoperative American Society of Anesthesiology grade and increased morbidity and mortality rates in patients undergoing spine surgery. J Neurosurg Spine 2011;14:470-4.

7. Zhang HQ, Li JS, Zhao SS, et al. Surgical management for thoracic spinal tuberculosis in the elderly: posterior only versus combined posterior and anterior approaches. Arch Orthop Trauma Surg 2012;132: 1717-23.

8. Xu Z, Wang X, Shen X, et al. Posterior only versus combined posterior and anterior approaches for lower lumbar tuberculous spondylitis with neurological deficits in the aged. Spinal Cord 2015;53:482-7.
9. Rawall S, Mohan K, Nene A. Posterior approach in thoracolumbar tuberculosis: a clinical and radiological review of 67 operated cases. Musculoskelet Surg 2013;97:67-75.

10. Hempelmann RG, Mater E, Schon R. Septic hematogenous lumbar spondylodiscitis in elderly patients with multiple risk factors: efficacy of posterior stabilization and interbody fusion with iliac crest bone graft. Eur Spine J 2010;19:1720-7.

11. Patil SS, Rawall S, Nagad P, Shial B, Pawar U, Nene AM. Outcome of single level instrumented posterior lumbar interbody fusion using corticocancellous laminectomy bone chips. Indian J Orthop 2011;45: 500-3.

12. Wang MY, Green BA, Shah S, Vanni S, Levi AD. Complications associated with lumbar stenosis surgery in patients older than 75 years of age. Neurosurg Focus 2003;14:e7.

13. Luo C, Wang X, Wu P, Ge L, Zhang H, Hu J. Singlestage transpedicular decompression, debridement, posterior instrumentation, and fusion for thoracic tuberculosis with kyphosis and spinal cord compression in aged individuals. Spine J 2016;16:154-62.

14. Younger EM, Chapman MW. Morbidity at bone graft donor sites. J Orthop Trauma 1989;3:192-5.

15. Patil S, Rawall S, Singh D, et al. Surgical patterns in osteoporotic vertebral compression fractures. Eur Spine J 2013;22:883-91. 